\title{
AVALIAÇÃO ERGONÔMICA DE CADEIRAS DE MADEIRA E DERIVADOS ${ }^{1}$
}

\author{
Amaury Paulo de Souza ${ }^{2}$, Patrícia Bhering Fialho ${ }^{3}$, Luciano José Minette ${ }^{4}$ e José de Castro Silva ${ }^{2}$
}

\begin{abstract}
RESUMO - Este trabalho teve como objetivo realizar a avaliação ergonômica de cadeiras de madeira e derivados fabricadas no Polo Moveleiro de Carmo do Cajuru, MG, visando à melhoria da qualidade ergonômica dos móveis, envolvendo aspectos de adaptação antropométrica, conforto e de segurança. A coleta de dados foi realizada em cadeiras fabricadas por indústrias associadas ao Sindicato das Indústrias do Mobiliário e de Artefatos de Madeira no Estado de Minas Gerais de Carmo do Cajuru - SINDIMOV, MG. Os critérios de conformidade foram definidos de acordo com os princípios de antropometria, ergonomia e os aspectos de segurança para o usuário. Os resultados das medições foram confrontados com recomendações e dados antropométricos existentes na literatura. Observou-se que os maiores problemas detectados nas cadeiras estavam relacionados às dimensões do assento. A altura dos assentos de todas as cadeiras foi superior aos valores recomendados na literatura. Os encostos não apresentaram problemas quanto à sua largura, porém necessitavam de adequação dos ângulos de inclinação em relação aos assentos. Outro problema encontrado foi a grande incidência de quinas e bordas retas que podem ocasionar injúrias aos usuários desses móveis.
\end{abstract}

Palavras-chave: Cadeiras, Móveis e Ergonomia.

\section{ERGONOMIC EVALUATION OF CHAIRS MADE OF WOOD AND ITS DERIVATIVES}

\begin{abstract}
This study aimed to carry out an ergonomic assessment of chairs and wooden products manufactured in the furniture cluster of Carmo do Cajuru, $M G$, and improve the ergonomic quality of furniture, involving aspects of anthropometric adaptation, comfort and safety. Data were collected from chairs manufactured by industries associated with the Union of Furniture Industries and Wooden Artifacts located in Carmo do Cajuru in the State of Minas Gerais - SINDIMOV, MG. Compliance criteria were defined according to the anthropometric principles, aspects of ergonomics and safety for the user. Measurements were compared with anthropometric data and recommendations in the literature. The major problem identified in the chairs were related to seat dimensions. Seat height of all chairs were above the literature recommendations. The back of the chairs showed no problems related width, however, adjustment of slope angles are required. Another problem was the high incidence of pointy corners and sharp edges that can cause injuries to users.
\end{abstract}

Keywords: Chairs, Furniture e Ergonomics.

\section{INTRODUÇÃO}

O uso da ergonomia vem-se tornando cada vez mais fator de competitividade na indústria moveleira, visto que é notório o aumento da procura dos consumidores por móveis que apresentam maior qualidade, segurança e conforto.
Dentro do Estado de Minas Gerais, um dos principais polos moveleiros é o de Carmo do Cajuru, caracterizado pela produção de uma tipologia variada de móveis, como: jogos de sala de jantar, estantes, racks, dormitórios e estofados. Entretanto, verifica-se que a ergonomia não é contemplada em muitos móveis fabricados nesse polo, ficando em primeiro plano apenas o aspecto estético do produto.

\footnotetext{
${ }^{1}$ Recebido em 01.04.2008 e aceito para publicação em 14.10.2009.

${ }^{2}$ Departamento de Engenharia Floresta, Universidade Federal de Viçosa - Vicosa, MG - Brasil E-mail: <amaury@ufv.br>e <jcastro@ufv.br>.

${ }^{3}$ Programa de Pós-Graduação em Ciência Florestal, UniversidadeFederal de Viçosa-Vicosa,MG-Brasi.E-mail: <patbhering@yahoo.com.br>. ${ }^{4}$ Departamento de Engenharia de Produção, Universidade Federal de Viçosa - Vicosa, MG - Brasil. E-mail: <minetti@ufv.br>.
} 
Segundo o Instituto Euvaldo Lodi (2003), a cadeira é um dos produtos mais fabricados em Carmo do Cajuru. A fabricação desse tipo de móvel tem como finalidade o uso doméstico, basicamente para uso em salas de jantar.

Segundo Costa (2005), os princípios ergonômicos utilizados em projetos de cadeiras são: segurança, postura, ângulos de conforto, dimensões adequadas para encosto e assento, entre outros, em contínua busca pelo bem-estar dos usuários.

No Brasil, não há normas técnicas direcionadas às cadeiras para uso residencial. Essa situação dificulta a elaboração de projetos e facilita a concorrência injusta com móveis de má qualidade disponíveis no mercado.

Segundo Panero e Zelnik (2002), uma das principais dificuldades em projetos de cadeiras é que o sentar-se é frequentemente visto como atividade estática, enquanto na verdade ela é dinâmica. Esse dinamismo acorre pelo fato de os indivíduos estarem em constante movimento e adotarem diversas posturas enquanto estão sentados. Dessa forma, é essencial que sejam utilizados dados antropométricos adequados, para se obterem as medidas e os espaços livres necessários para a movimentação do usuário.

Para Silva et al. (2006), o levantamento antropométrico de uma população é muito relevante em estudos ergonômicos, uma vez que fornece subsídios para que determinada atividade realizada não se torne fator de danos à saúde e desconforto.

Segundo Fialho et al. (2006), existem poucas publicações de dados antropométricos dinâmicos e funcionais da população brasileira, o que dificulta a realização de projetos adaptados à população.
A aplicação da ergonomia em cadeiras mostra-se importante, uma vez que esse mobiliário é utilizado por diferentes pessoas, com idades, tamanhos e comportamentos distintos, proporcionando maior segurança, conforto e bem-estar.

Este trabalho teve como objetivo realizar uma avaliação ergonômica de cadeiras de madeira e derivados fabricadas no Polo Moveleiro de Carmo do Cajuru, MG, visando à melhoria da qualidade ergonômica desses móveis envolvendo aspectos de adaptação antropométrica, de conforto e de segurança.

\section{MATERIAL E MÉTODOS}

A coleta de dados para o desenvolvimento deste trabalho foi realizada em 12 indústrias previamente escolhidas pelo SINDIMOV-MG - Sindicato das Indústrias do Mobiliário e de Artefatos de Madeira no Estado de Minas Gerais de Carmo do Cajuru, MG.

Os dados foram coletados de 13 modelos de cadeiras de uso doméstico mais vendidas de cada indústria, visando abranger maior representatividade desses móveis disponíveis no mercado.

Os critérios de conformidade ergonômica foram definidos de acordo com os princípios ergonômicos de antropometria e os aspectos de segurança. Os principais itens de avaliação ergonômica utilizados neste estudo foram: dimensões (comprimento, largura e altura), inclinação entre o assento e o encosto, tipo de borda anterior do assento e a presença de quinas e bordas retas.

As dimensões dos móveis foram obtidas por meio de medições diretas, utilizando-se instrumentos como goniômetro, trenas graduadas e fitas métricas. As dimensões avaliadas nas cadeiras estão representadas na Figura 1. Largura do encosto

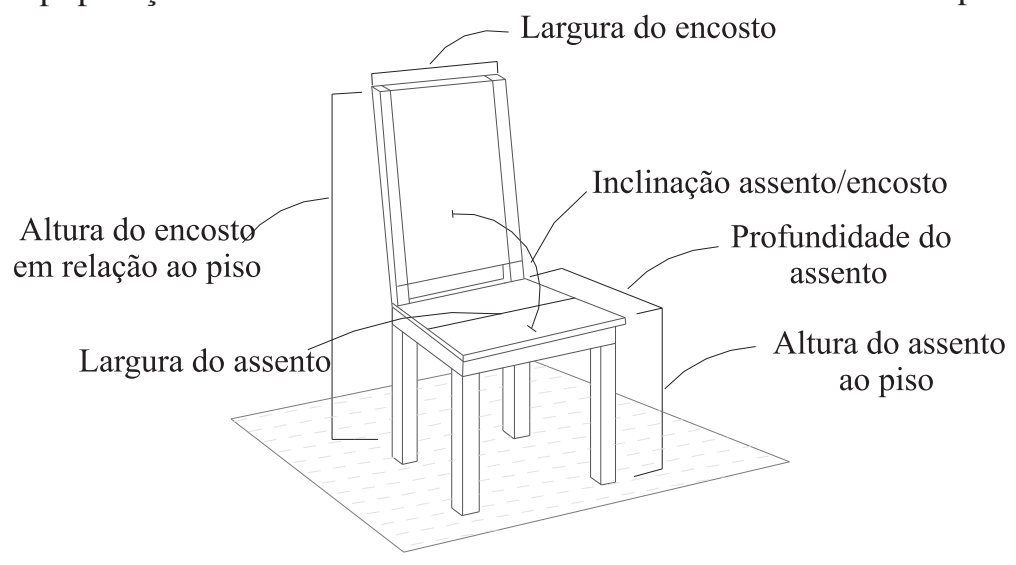

Figura 1 - Dimensões avaliadas em cadeiras.

Figure 1 - Evaluated chair dimensions.

R. Árvore, Viçosa-MG, v.34, n.1, p.157-164, 2010 


\section{RESULTADOS}

\subsection{Dimensões}

Entre as amostras de assentos das cadeiras analisadas, verificou-se que as suas alturas até o piso variaram de $45,7 \mathrm{~cm}$ a $52,0 \mathrm{~cm}$, numa amplitude de 6,3 $\mathrm{cm}$, e as profundidades, entre $35,2 \mathrm{~cm}$ e $45,0 \mathrm{~cm}$, numa amplitude de $9,8 \mathrm{~cm}$. Já as larguras desses assentos variaram entre $38,0 \mathrm{~cm}$ e $49,0 \mathrm{~cm}$, equivalendo a uma amplitude de $11,0 \mathrm{~cm}$.

Quanto aos encostos das cadeiras, verificou-se que as suas alturas em relação ao piso variaram entre $88,5 \mathrm{~cm}$ e $124,0 \mathrm{~cm}$, com amplitude de $35,5 \mathrm{~cm}$, e as suas larguras variaram de $30,0 \mathrm{~cm}$ a $49,0 \mathrm{~cm}$, com amplitude de $19,0 \mathrm{~cm}$.

No tocante às inclinações do encosto em relação ao assento, observou-se que essa medida variou entre $90^{\circ}$ e $110^{\circ}$, com amplitude de $20^{\circ}$.

\subsection{Conforto}

Do total de cadeiras analisadas, verificou-se que $84,6 \%$ apresentaram algum tipo de estofamento, sendo $100 \%$ destes feitos de espuma.

\subsection{Aspectos de segurança}

Entre as cadeiras analisadas, 38,5\% apresentaram quinas retas e $23,7 \%$, bordas retas. Verificou-se, ainda, que $23,0 \%$ não possuíam bordas anteriores de assentos arredondadas.

\section{DISCUSSÃO}

\subsection{Dimensões}

Os dados obtidos na coleta foram confrontados com as recomendações do Instituto de Biomecânica de Valência - IBV (1992), de Panero e Zelnik (2002), Gomes (2003) e com dados antropométricos do INT (1995).

Para efeito de comparação, as dimensões dos móveis atenderam às principais variáveis antropométricas, como: altura popliteal; largura do quadril, profundidade nádegapoplíteal para a pessoa sentada, inclinação de conforto do tronco e largura do tórax.

\subsubsection{Assento}

\subsubsection{Altura do assento em relação ao piso}

A Figura 2 apresenta os valores medidos das alturas das cadeiras coletados nos móveis e valores recomendados por Panero e Zelnik (2002) (mínima de
40,6 cm e máxima de 41,3 cm), por Gomes (2003) (40,0 $\mathrm{cm}$ ) e pelo Instituto de Biomecânica de Valência (1992) $(41,0 \mathrm{~cm})$. A Figura 2 apresenta, ainda, o valor referente ao dado antropométrico do INT (1995), relativo à altura popliteal de mulheres com percentil $50(41,0 \mathrm{~cm})$.

Ao comparar esses valores, verificou-se que $100 \%$ das cadeiras apresentaram alturas dos assentos superiores às recomendações da literatura e ao dado antropométrico do INT (1995).

A partir desses resultados, constatou-se que essas elevadas alturas dos assentos podem causar diversos problemas na saúde dos usuários. Como citaram Moraes e Pequini (2000), a discrepância na altura do assento pode acarretar problemas de circulação sanguínea e varizes.

Além dos problemas ergonômicos, as elevadas alturas dos assentos acarretam, ainda, desperdício da matéria-prima utilizada na fabricação desses móveis.

De acordo com o IBV (1992), a altura do assento de uma cadeira deve permitir que os pés do usuário se apoiem firmemente no piso ou em um apoio. $\mathrm{O}$ assento não deve ser excessivamente baixo e nem alto, uma vez que o assento muito alto pode provocar pressão nos músculos dos usuários mais baixos e o muito baixo, provocar pressão nas nádegas dos usuários mais altos.

Acrescentando a essa posição, Panero e Zelnik (2002) afirmaram que, se a altura do assento for muito baixa, os pés podem perder estabilidade pelo fato de as pernas terem de ficar estendidas para frente. Uma pessoa mais alta, no entanto, sentir-se-á mais confortável usando uma cadeira com assento baixo do que uma pessoa baixa usando uma cadeira com assento muito alto.

\subsubsection{Profundidade do assento}

A Figura 3 apresenta os valores medidos das profundidades das cadeiras coletados nos móveis e valores recomendados por Panero e Zelnik (2002) (mínima de 39,4 cm e máxima de 40,6 cm), pelo Instituto de Biomecânica de Valência (1992) (mínima de 40,0 cm e máxima de 42,0 cm). Apresenta, também, os valores referentes aos dados antropométricos do INT (1995), relativos ao comprimento nádega-popliteal de mulheres com percentil $50(45,7 \mathrm{~cm})$ e percentil $5(40,5 \mathrm{~cm})$.

R. Árvore, Viçosa-MG, v.34, n.1, p.157-164, 2010 


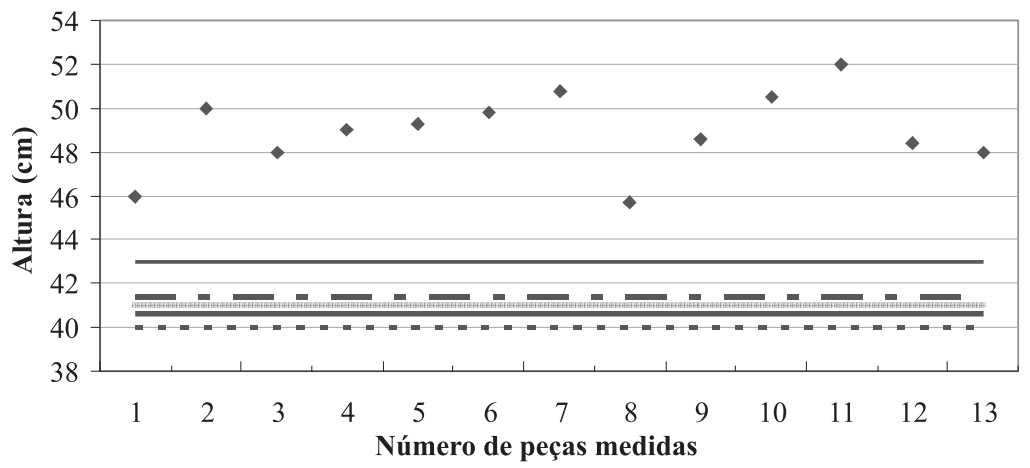

- Valores medidos

_- Gomes (2003) e IBV (1992 - altura máxima de 43,0 cm

- - Panero e Zelnik (2002) - altura máxima de 41,3 cm

Com base no dado antropométrico do INT (1995) - altura popliteal de mulheres com percentil 50 e valor mínimo recomendado pelo IBV (1992) - 41,0cm Panero e Zelnik (2002) - altura mínima de 40,6 cm

- - - Gomes (2003) - altura mínima de 40,0 cm

Figura 2 - Comparação entre os valores das alturas dos assentos e os valores referentes ao dado antropométrico do INT (1995) e as recomendações de Panero e Zelnik (2002), IBV (1992) e Gomes (2003).

Figure 2 - Comparison between seat heights and anthropometric data by INT (1995) and recommendations by Panero and Zelnik (2002), IBV (1992) and Gomes (2003).

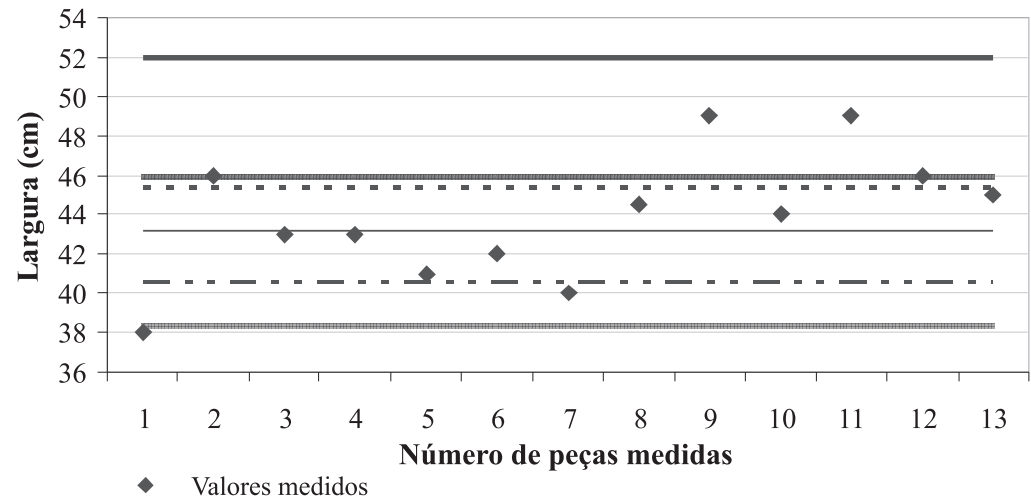

- Valores medidos

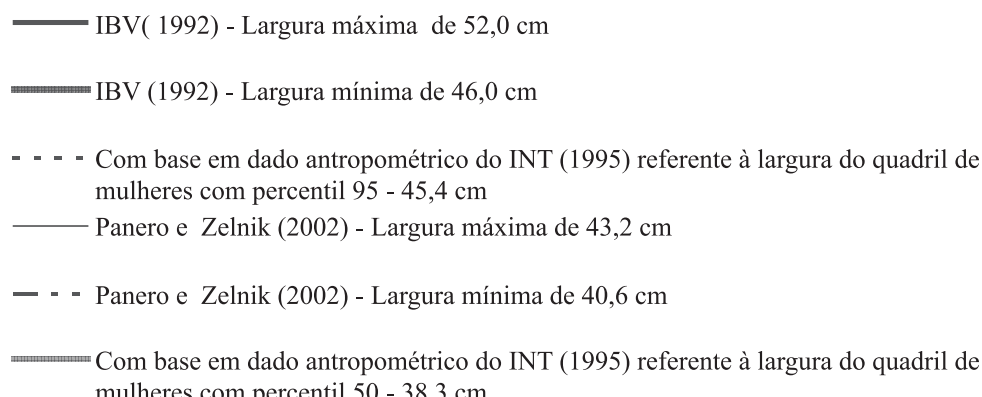

Figura 3 - Comparação entre os valores das profundidades dos assentos e os valores referentes aos dados antropométricos do INT (1995) e as recomendações de Panero e Zelnik (2002) e do IBV (1992).

Figure 3 - Comparison between seat depths and reference anthropometric data by INT (1995) and recommendations by Panero and Zelnik (2002) and IBV (1992).

R. Árvore, Viçosa-MG, v.34, n.1, p.157-164, 2010 
Ao comparar os valores medidos neste trabalho com os dados antropométricos do INT (1995), observou-se que $38,5 \%$ das cadeiras não atenderam ao valor relacionado ao percentil 5, uma vez que apresentaram profundidades inferiores a 40,5 cm. Quando comparados esses mesmos valores com o percentil 50, verificou-se que $100 \%$ das cadeiras não atenderam a esse dado, uma vez que apresentaram profundidades dos assentos inferiores a $45,7 \mathrm{~cm}$.

Também, neste trabalho se, observou que 76,9\% das cadeiras não atenderam às recomendações de Panero e Zelnik (2002), uma vez que não apresentaram profundidades dos assentos entre os valores mínimo e máximo estabelecidos por esses autores, e 38,5\% das cadeiras mostraram valores de larguras de acordo com as recomendações do IBV (1995).

Segundo Panero e Zelnik (2002), os assentos com muita profundidade podem causar compressão nos tecidos e gerar desconforto ao usuário. Os assentos com pouca profundidade podem causar falta de suporte na parte inferior das coxas, dando a sensação de que o usuário está caindo do assento.
Para o IBV (1992), o excesso na profundidade do assento pode fazer, ainda, que os usuários mais baixos não usem o encosto para evitar a pressão nas pernas.

\subsubsection{Largura do assento}

A Figura 4 apresenta os valores medidos das larguras das cadeiras coletados nos móveis e valores recomendados por Panero e Zelnik (2002) (mínima de $40,6 \mathrm{~cm}$ e máxima de $43,2 \mathrm{~cm}$ ) e pelo Instituto de Biomecânica de Valência (1992) (mínima de 46,0 cm e máxima de 52,0 cm). A Figura 4 apresenta, ainda, os valores referentes aos dados antropométricos do INT (1995), relativo à largura do quadril de mulheres com percentil $50(38,3 \mathrm{~cm})$ e à altura popliteal de mulheres com percentil $95(45,4 \mathrm{~cm})$.

Ao comparar esses valores, verificou-se que 30,8\% das cadeiras apresentaram largura do assento entre as recomendações de Panero e Zelnik (2002). Observou-se, também, que $92,3 \%$ das cadeiras atenderam ao valor referente ao dado antropométrico do INT (1995) para largura do quadril de mulheres com percentil 50, uma vez que apresentaram valores das larguras dos assentos

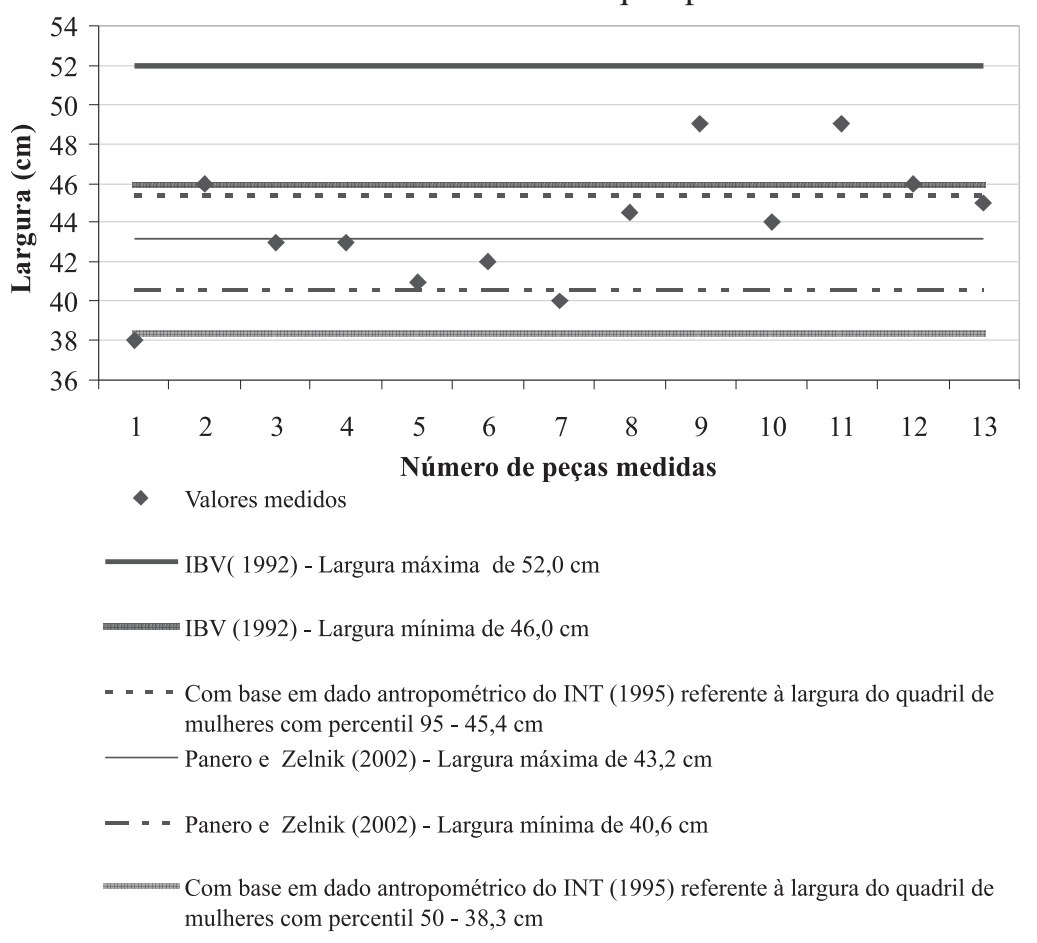

Figura 4 - Comparação entre os valores das larguras dos assentos e os valores referentes aos dados antropométricos do INT (1995), as recomendações de Panero e Zelnik (2002) e do IBV (1992).

Figure 4-Comparison between seat widths and reference anthropometric data by INT (1995), recommendations by Panero and Zelnik (2002) and IBV (1992). 
superiores a esse dado antropométrico. Verificou-se, ainda, que $30,8 \%$ das cadeiras atenderam ao valor referente ao dado antropométrico do INT (1995) para largura do quadril de mulheres com percentil 95, em que exibiram valores das larguras dos assentos superiores a esse dado antropométrico e valores entre as recomendações do IBV (1992).

\subsubsection{Encosto}

Para Panero e Zelnik (2002), “o encosto da cadeira deveria permitir o apoio da região lombar". Observou-se, no entanto, que faltam publicações de dados antropométricos sobre essa região, que poderiam auxiliar na comparação com os dados adquiridos na coleta de dados.

Na realização deste trabalho foram encontradas somente duas recomendações relacionadas com encostos, referentes à sua largura e á altura até o piso.

\subsubsection{Altura de encosto em relação ao piso}

Segundo Panero e Zelnik (2002), para cadeiras de múltiplo uso, com curto período de tempo, a mínima altura do encosto ao piso deveria ser de $78,7 \mathrm{~cm}$ e a máxima, de $83,8 \mathrm{~cm}$.

Quando comparados os valores referentes à altura dos encostos coletados no trabalho (que variaram entre 88,5 e 124 cm) com as recomendações mínimas e máximas de Panero e Zelnik (2002), observou-se que $100 \%$ das cadeiras apresentaram altura de encosto superior àquelas recomendadas por esses autores.

\subsubsection{Largura do encosto}

Ao comparar os valores das larguras dos encostos coletados no trabalho com o valor referente ao dado antropométrico do INT (1995), relativo à largura do tórax entre as axilas, para homens com percentil 50 $(34,9 \mathrm{~cm})$, observou se que $92,3 \%$ dos encostos atenderam a esse valor, uma vez que apresentavam largura superior a $34,9 \mathrm{~cm}$.

Quando comparados esses mesmos valores de larguras dos encostos com o dado antropométrico, relativo à largura do tórax entre as axilas de homens com percentil $95(39,1 \mathrm{~cm})$, observou-se que $62,2 \%$ dos encostos estavam de acordo com esse dado, uma vez que apresentaram largura superior a $39,1 \mathrm{~cm}$.

R. Árvore, Viçosa-MG, v.34, n.1, p.157-164, 2010

\subsubsection{Inclinação do encosto}

Conforme Grandjean (1998), quando o ângulo entre o assento e o encosto for a partir de $90^{\circ}$, tem-se diminuição na pressão dos discos intervertebrais e no trabalho estático da musculatura das costas.

Panero e Zelnik (2002) recomendaram que os ângulos de inclinações entre o assento e o encosto das cadeiras de múltiplo uso deveriam ser de $105^{\circ}$, pois ângulos menores que esse valor poderiam causar desconforto ao usuário; ângulos maiores poderiam prejudicar os movimentos dos usuários para sentar-se e levantar-se das cadeiras. Gomes (2003) recomendou que esse ângulo de inclinação para conforto de cadeiras residenciais estivesse entre $95^{\circ}$ e $105^{\circ}$. Já o IBV (1992) recomendou que ele fosse entre $95^{\circ} \mathrm{e} 105^{\circ}$. Ainda segundo o IBV, ângulos superiores a $110^{\circ}$ são reservados para cadeiras de descanso.

Através do gráfico da Figura 5, verificou-se que nenhum dos encostos analisados estava de acordo com as recomendações de Panero e Zelnik (2002), uma vez que não apresentaram inclinações iguais a $105^{\circ}$. Verificou-se, também, que 53,8\% desses encostos estavam de acordo com Gomes (2003), apresentando inclinações entre os ângulos máximos e mínimos recomendados por esse autor, e 46,2\% atendiam às recomendações do IBV (1992).

\subsection{Conforto}

A presença do estofamento em $84,6 \%$ das cadeiras foi considerada neste trabalho como ponto positivo, apesar de a sensação de conforto ser subjetiva e individual. Paschoarelli e Silva (1996) citaram que a cadeira almofadada influencia a percepção do usuário, proporcionando solução de maior comodidade.

Entre as cadeiras que não apresentavam estofamento, verificou-se que $50 \%$ apresentavam assentos com relevos marcados. Segundo o IBV (1992), os assentos sem estofamento não deveriam apresentar relevos marcados, uma vez que podem dificultar a mudança de postura.

\subsection{Aspectos de segurança}

Os aspectos de segurança foram tratados neste trabalho, enfatizando a presença de quinas e bordas retas.

Neste trabalho, o fato de $38,5 \%$ das cadeiras avaliadas apresentarem quinas retas, e $23,7 \%$ das bordas retas foi considerado negativo, uma vez que quinas e bordas retas podem causar acidentes domésticos, prejudicando os usuários desse tipo de mobiliário. 


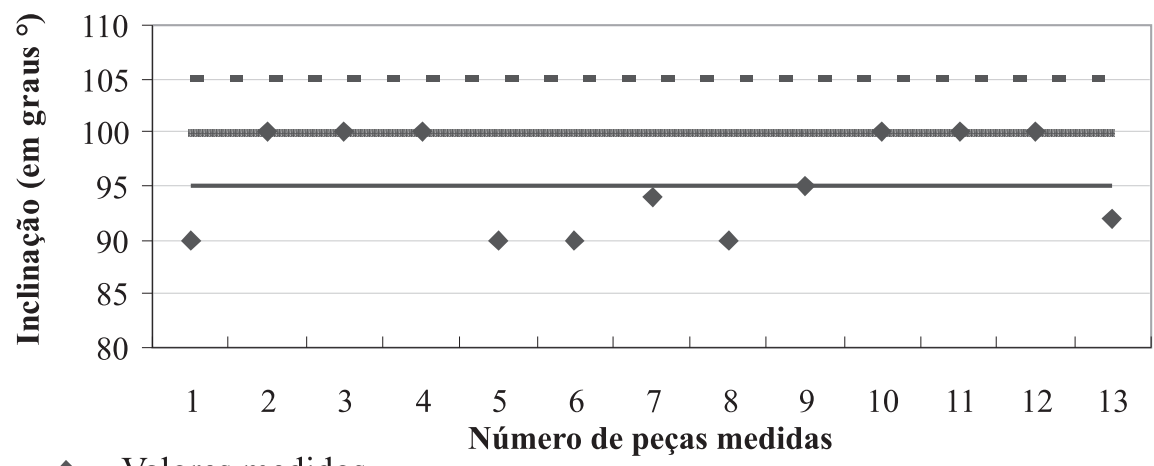

- Valores medidos

Número de peças medidas

— Recomendação mínima de Gomes (2003) - 95 graus

——_- Recomendação mínima IBV (1992) - 100 graus

- - - Recomendação máxima de Gomes (2003) e IBV (1992) e recomendação de Panero e Zelnik (2002) - 105 graus

Figura 5 - Comparações entre os valores das inclinações dos encostos em relação aos assentos e as recomendações de Panero e Zelnik (2002) e Gomes (2003).

Figure 5 - Comparisons between seat back slopes and recommendations by Panero and Zelnik (2002), Gomes (2003) and IBV (1992).

Em adição a isso, o fato de $23,0 \%$ das cadeiras não apresentarem bordas anteriores de assentos arredondadas não foi considerado satisfatório, uma vez que, como citaram Panero e Zelnik (2002), do ponto de vista ergonômico bordas retas de assentos podem causar, além de desconforto, compressão dos tecidos internos e, consequentemente, diminuição da circulação sanguínea na parte inferior da coxa.

\section{CONCLUSÕES}

Constatou-se que os maiores problemas detectados nas cadeiras estavam relacionados à profundidade e altura do assento. Todas as cadeiras apresentaram alturas do assento em desacordo com aos valores recomendados pela literatura. Os encostos não mostraram problemas quanto à sua largura, porém necessitavam de adequação dos ângulos de inclinação em relação aos assentos. Verificou-se, ainda, a grande incidência de quinas e bordas retas, que podem ocasionar acidentes aos usuários desses móveis. O uso de dimensões superestimadas nas cadeiras poderia ser evitado, por exemplo: reduzindo a altura dos assentos das cadeiras avaliadas, isso minimizaria os problemas ergonômicos como o desconforto e, ainda, diminuiria o custo final do produto.

\section{AGRADECIMENTOS}

À Fundação de Amparo à Pesquisa do Estado de Minas Gerais - FAPEMIG, pelo apoio com bolsas e suporte financeiro a este projeto; ao Conselho Nacional de Desenvolvimento Científico e Tecnológico-CNPq, pelo oferecimento da bolsa de produtividade em pesquisa ao primeiro e terceiro autores e pela bolsa de doutorado à segunda autora; e ao Sindicato das Indústrias do Mobiliário e de Artefatos de Madeira no Estado de Minas Gerais - SINDIMOV de Carmo do Cajuru, pelo apoio logístico a este projeto.

\section{REFERENCIAS}

COSTA, F. F. Avaliação da conformidade ergonômica de cadeiras residenciais de visando ao redesign do APL de Ubá e região, MG. 2005. 74f. Dissertação (Mestrado em Ciências Florestais) - Universidade Federal de Viçosa, Viçosa, MG, 2005.

FIALHO, P. B. et al. Avaliação ergonômica de cadeiras residenciais fabricadas no pólo moveleiro de Ubá, MG. Revista Árvore, v.31, n.5, p.887896, 2007.

R. Árvore, Viçosa-MG, v.34, n.1, p.157-164, 2010 
GOMES, J. F. Ergonomia do objeto: sistema técnico de leitura ergonômica. São Paulo:

Escrituras, 2003.

GRANDJEAN, E. O assento de trabalho. In: Manual de ergonomia: adaptando o trabalho ao homem. 4.ed. Porto Alegre: Bookman, 1998. p.60-72.

INSTITUTO EUVALDO LODI - IEL. Diagnóstico das indústrias moveleiras de Carmo do Cajuru. Belo Horizonte: 2003. 80p.

PASCHOARELLI, L. C.; SILVA, J. C. P.

Considerações no design de carteiras escolares da pré-escola para uma adequada postura de trabalho. In: CONGRESSO BRASILEIRO DE PESQUISA E DESENVOLVIMENTO EM DESIGN, 2., 1996, Belo

Horizonte. Anais... Belo Horizonte: 1996. p.33-43.
INSTITUTO NACIONAL DE TECNOLOGIA INT. Manual de aplicação dos dados antropométricos - Ergokit. Rio de Janeiro: 1995.

MORAES, A.; PEQUINI, S. M. Ergodesign para terminais informatizados. Rio de Janeiro: 2AB, 2000. 124p.

PANERO, J.; ZELNIK, M.

Dimensionamento humano para espaços interiores. Barcelona: Gustavo Gili, 2002.320p.

SILVA, K. R. et al. Avaliação antropométrica de trabalhadores em indústrias do pólo moveleiro de Ubá, MG. Revista Árvore, v.30, n.4, p.613-618, 2006. 